\title{
Erratum to: Autologous blood injection for treatment of chronic recurrent TMJ dislocation: is it successful? Is it safe enough? A systematic review
}

\author{
Payam Varedi $^{1,2} \cdot$ Behnam Bohluli ${ }^{2,3}$
}

Published online: 26 May 2015

(C) Springer-Verlag Berlin Heidelberg 2015

Erratum to: Oral and Maxillofacial Surgery

DOI 10.1007/s10006-015-0500-y

The original version of this manuscript contained multiple mistakes in the arrangements and abbreviations of Table 1. Below is the correct Table 1.

Behnam Bohluli

bbohluli@yahoo.com

Payam Varedi

payam.varedi@yahoo.com

1 Department of Oral and Maxillofacial Surgery, Faculty of Dentistry, Qazvin University of Medical Sciences, Qazvin, Iran

2 Craniomaxillofacial Research Center, Department of Oral and Maxillofacial Surgery, Shariati Hospital, Tehran University of Medical Sciences, Tehran, Iran

3 Department of Oral and Maxillofacial Surgery, Buali Hospital, Islamic Azad University of Medical Sciences, Tehran, Iran 


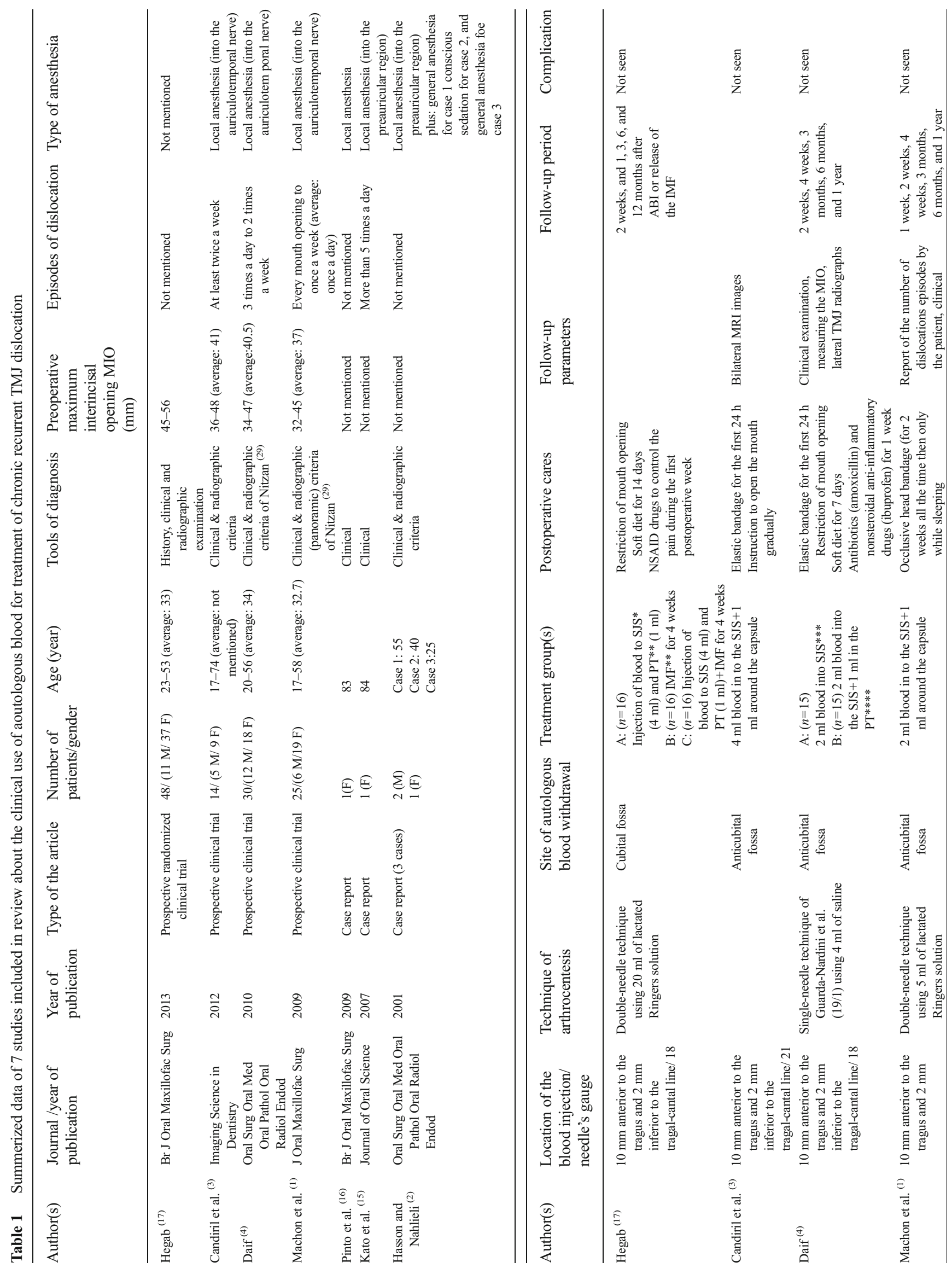




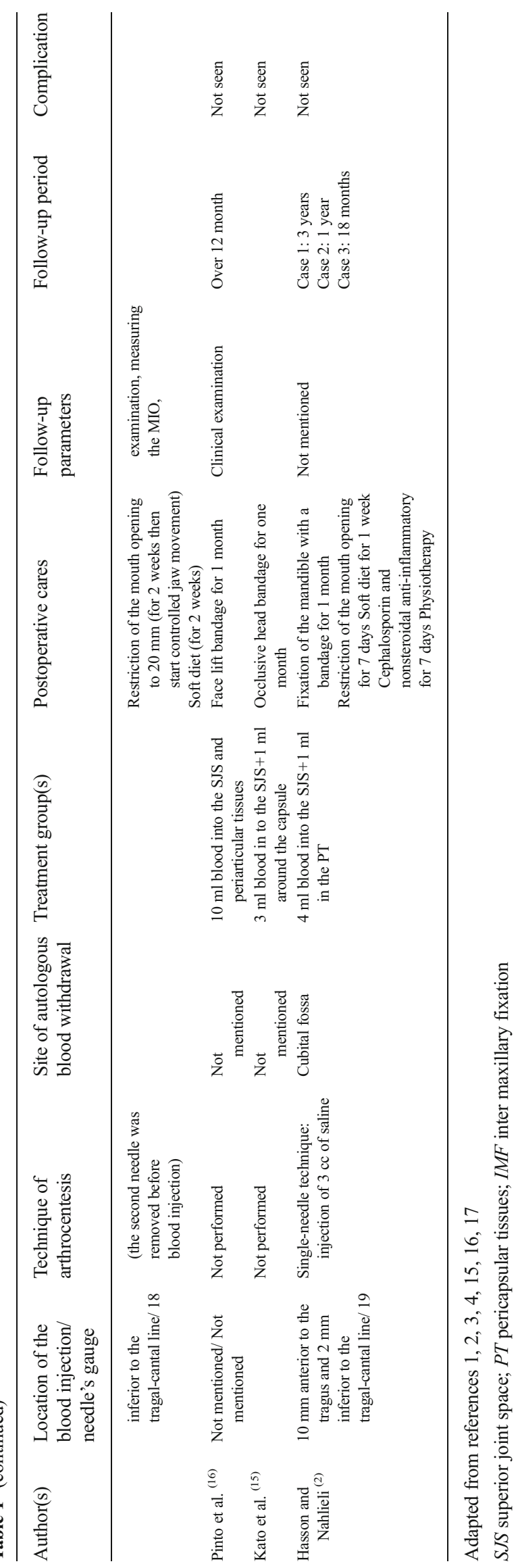

